\title{
Modulación del Estado de Óxido-Reducción por Peróxido de Hidrógeno en la Etapa de Maduración Ovocitaria: Efecto sobre el Desarrollo Embrionario en Bovinos
}

\author{
Modulation of Redox State by Hydrogen Peroxide in the Stage \\ of Oocyte Maturation: Effect on Embryonic Development in Cattle
}

\author{
Pía Loren*; Carolina Cheuquemán*; Jennie Risopatrón "***; Ricardo Felmer",***; \\ María E. Arias ${ }^{* * * * *}$ \& Raúl Sánchez ${ }^{*, * * * *}$
}

LOREN, P.; CHEUQUEMÁN, C.; RISOPATRón, J.; FELMER, R.; ARIAS, M. E. \& SÁNCHEZ, R. Modulación del estado de óxido-reducción por peróxido de hidrógeno en la etapa de maduración ovocitaria: efecto sobre el desarrollo embrionario en bovinos. Int. J. Morphol., 34(2):431-435, 2016.

RESUMEN: El estrés oxidativo es definido como un desbalance entre la producción de oxidantes y antioxidantes. La inducción de tolerancia a estrés en los ovocitos conllevaría a un mejor desarrollo embrionario. En bovinos, la incubación de ovocitos maduros con diferentes estresores (térmicos, alta presión hidrostática, oxidativos) incrementaría la tasa de generación de blastocitos. Este estudio evalúa el efecto de la modulación del estado redox incrementando el estrés oxidativo con $\mathrm{H}_{2} \mathrm{O}_{2}$ en ovocitos maduros bajo condiciones de cultivo in vitro y su efecto sobre el potencial de desarrollo embrionario. Para ello, ovocitos procedentes de ovarios de matadero fueron madurados en medio TCM-199 suplementado durante $22-23 \mathrm{~h}$, a 38,5 ${ }^{\circ} \mathrm{C}, 5 \% \mathrm{CO}_{2}$ y humedad a saturación. Al final de las $22-23$ h se incubaron los ovocitos maduros con 0, 50, 100 y $200 \mu \mathrm{M} \mathrm{H}_{2} \mathrm{O}_{2}$. La fecundación in vitro se realizó co-incubando los ovocitos durante $18 \mathrm{~h}$ con una concentración final de $1 \times 10^{6}$ espermatozoides $/ \mathrm{mL}$. Los presuntos cigotos fueron denudados y cultivados en medio KSOM- $0,4 \%$ BSA a $38,5{ }^{\circ} \mathrm{C}$ en atmósfera de baja tensión de $\mathrm{O}_{2}\left(5 \% \mathrm{O}_{2}, 5 \% \mathrm{CO}_{2}\right.$ y $\left.90 \% \mathrm{~N}_{2}\right)$ y humedad a saturación. El estrés oxidativo inducido con $\mathrm{H}_{2} \mathrm{O}_{2}$ a una concentración de 50 y $100 \mu \mathrm{M}$ produce una tasa de división de los embriones similar al control (88,7 \%, 83,2 \% y 86,4 \% respectivamente, $\mathrm{p}>0,05)$, disminuyendo significativamente al utilizar una concentración de $200 \mu \mathrm{M}(58,8 \%, \mathrm{p}<0,05)$. Asimismo, $\mathrm{H}_{2} \mathrm{O}_{2}$ causó un efecto similar en la tasa de blastocitos con $50 \mu \mathrm{M}(20,4 \%$ vs. $25,8 \%$ control, p>0.05) pero disminuyó significativamente con 100 y $200 \mu \mathrm{M}(10,7 \%$ y 3,3\% respectivamente, p<0,05). Es posible, que estos embriones resistentes al estrés oxidativo puedan tener una mayor sobrevida durante los procesos de criopreservación que generan altos niveles de especies reactivas de oxígeno en los embriones.

PALABRAS CLAVE: Fecundación in vitro; Estrés oxidativo; Tolerancia a estrés; Especies reactivas de oxígeno.

\section{INTRODUCCIÓN}

Durante los últimos años se ha intentado mejorar la calidad de los embriones generados en procedimientos in vitro. Sin embargo, las condiciones de cultivo in vitro no son similares a las condiciones de cultivo in vivo y pueden representar una fuente de estrés oxidativo para los embriones generados mediante procedimientos in vitro (Agarwal et al., 2006). El estrés oxidativo puede ser definido como un desbalance entre las moléculas oxidantes y los antioxidantes, lo que genera un estado de estrés en la célula (Ma, 2010). En el metabolismo celular, las especies reactivas de oxigeno (EROs) como $\mathrm{O}_{2} \bullet-, \mathrm{H}_{2} \mathrm{O}_{2}$ y $\bullet \mathrm{OH}-$ son generadas como subproductos de la respiración celular, específicamente en la cadena transportadora de electrones (Liu et al., 2002). Existe una dualidad en los efectos que generan las EROs, son necesarias en procesos fisiológicos, como por ejemplo en el fluido folicular para una adecuada formación y calidad del embrión (Das et al., 2006), maduración de ovocitos (Morado et al., 2009), en los espermatozoides modulan la capacitación, fosforilación de proteínas y reacción acrosoma (Roy $\&$ Atreja, 2008). Pero, en procesos patológicos los niveles incrementados de EROs pueden causar alteraciones en el desarrollo embrionario, apoptosis y arresto embrionario

* Centro de Biotecnología de la Reproducción (BIOREN-CEBIOR), Facultad de Medicina, Universidad de La Frontera, Temuco, Chile.

** Departamento de Ciencias Básicas, Facultad de Medicina, Universidad de La Frontera, Temuco, Chile.

*** Departamento de Ciencias Agropecuarias y Recursos Naturales, Facultad de Ciencias Agropecuarias y Forestales, Universidad de La Frontera, Temuco, Chile.

***** Departamento de Ciencias Preclínicas, Facultad de Medicina, Universidad de La Frontera, Temuco, Chile.

Proyecto FONDECYT 1130888, CONICYT, Gobierno de Chile. 
(Goto et al., 1993). Sin embargo, en embriones bovinos la incubación de ovocitos con 50-100 $\mathrm{MM} \mathrm{H}_{2} \mathrm{O}_{2}$ resulta en un incremento en el porcentaje de blastocitos $(47,3 \%)$ en comparación con el control (31,8\%), mientras que el número de células apoptóticas fue inversamente relacionado con la concentración de $\mathrm{H}_{2} \mathrm{O}_{2}$ (Vandaele et al., 2010). De la misma manera, la fecundación in vitro de ovocitos con espermatozoides expuestos a $200 \mu \mathrm{M} \mathrm{H}_{2} \mathrm{O}_{2}$ incrementó la fecundación, la división y la tasa de generación de blastocitos (Rahman et al., 2012).

El nuevo concepto de que la inducción de tolerancia a estrés en los ovocitos conllevaría a un mejor desarrollo embrionario, ha creado una nueva oportunidad para estudiar estos factores. De acuerdo a esto, el objetivo de este estudio es evaluar si la inducción de estrés oxidativo después de la finalización de la maduración de ovocitos a través de la exposición a $\mathrm{H}_{2} \mathrm{O}_{2}$ genera tolerancia a estrés, mejorando la calidad y el potencial de desarrollo de embriones bovinos durante el cultivo in vitro.

\section{MATERIAL Y MÉTODO}

Reactivos: Todos los reactivos utilizados fueron obtenidos de Sigma (St. Louis, MO, USA).

Selección y maduración de ovocitos: Ovarios bovinos fueron colectados de hembras de un matadero local (Frigorífico Temuco, Chile) en una solución $\mathrm{NaCl} 0,9 \%$ (p/v) suplementado con $1 \%(\mathrm{v} / \mathrm{v})$ penicilina/estreptomicina a $37{ }^{\circ} \mathrm{C}$. En el laboratorio, los ovarios fueron lavados dos veces en una solución $\mathrm{NaCl} \mathrm{0,9 \%} \mathrm{(p/v).} \mathrm{Los} \mathrm{ovocitos} \mathrm{fueron} \mathrm{aspira-}$ dos desde folículos entre $2-8 \mathrm{~mm}$ de diámetro utilizando una aguja calibre 18 acoplada a una jeringa de $10 \mathrm{~mL}$, seleccionados de acuerdo al criterio de citoplasma homogéneo y al menos cuatro capas de células del cúmulo. Los ovocitos seleccionados fueron cultivados en grupos de 4050 en $500 \mu \mathrm{L}$ de TCM-199 suplementado con $10 \%$ (v/v) de suero bovino fetal inactivado (Hyclone Laboratories, Inc., UT, USA), 0,2 mM piruvato de sodio, $25 \mu \mathrm{g} / \mathrm{mL}$ sulfato de gentamicina, $6 \mathrm{mg} / \mathrm{mL}$ hormona FSH (Sioux Biochemical, Inc., Sioux City, IA, USA), $6 \mu \mathrm{g} / \mathrm{mL}$ hormona LH (Sioux Biochemical) y $1 \mathrm{mg} / \mathrm{mL}$ estradiol por $22-23 \mathrm{~h}$ a $38.5^{\circ} \mathrm{C}, 5$ $\% \mathrm{CO}_{2}$ y humedad a saturación.

Preparación de espermatozoides: Semen bovino criopreservado con fertilidad probada fue descongelado en un baño termorregulado a $37^{\circ} \mathrm{C}$ durante 45 s. Posteriormente, fue separado a través de un gradiente discontinuo de Percoll $^{\circledR}$ (45 y 90 \% (v/v); Pharmacia, USA) y lavados con TALP-Sperm, suplementado con 0,2 mM piruvato de sodio,
$6 \mathrm{mg} / \mathrm{mL}$ albumina sérica bovina (BSA) libre de ácidos grasos y $10 \mu \mathrm{g} / \mathrm{mL}$ sulfato de Gentamicina. La concentración final fue ajustada para fecundar con $1 \times 10^{6}$ espermatozoides/mL con TALP-IVF suplementado con 0,2 $\mathrm{mM}$ piruvato de sodio, $6 \mathrm{mg} / \mathrm{mL}$ BSA y $10 \mu \mathrm{g} / \mathrm{mL}$ sulfato de Gentamicina.

Fecundación in vitro: Los ovocitos maduros fueron lavados en medio TALP-Sperm tres veces y una vez en TALPIVF y luego depositados en la placa de fecundación. Se adicionó a esta placa $2 \mu \mathrm{g} / \mathrm{mL}$ heparina, $80 \mu \mathrm{M}$ penicilamina, $40 \mu \mathrm{M}$ hipotaurina, $10 \mu \mathrm{M}$ epinefrina y en último lugar los espermatozoides previamente seleccionados en el gradiente de densidad. Los ovocitos fueron co-incubados con los espermatozoides durante $18 \mathrm{~h}$ a $38,5^{\circ} \mathrm{C}, 5 \% \mathrm{CO}_{2}$ y humedad a saturación.

Cultivo in vitro: Luego de la fecundación, los presuntos cigotos fueron vortexeados para remover las células del cúmulo y transferidos a gotas de $50 \mu \mathrm{L}$ de medio KSOM (EmbryoMax, Millipore Corp, Billerica, MA, USA) bajo aceite mineral en grupos de 25-30 cigotos en una atmósfera de baja tensión de oxígeno $\left(5 \% \mathrm{CO}_{2}, 5 \% \mathrm{O}_{2}\right.$ y $95 \%$ N2), $38,5^{\circ} \mathrm{C}$ y humedad a saturación. A las $72 \mathrm{~h}$ del cultivo, se suplementó con suero bovino fetal $5 \%(\mathrm{v} / \mathrm{v})$.

Evaluación de desarrollo embrionario: Se evaluó la tasa de división de los embriones cultivados in vitro $72 \mathrm{~h}$ post inicio del cultivo, así como la tasa de generación de blastocitos al día 7 post inicio de cultivo embrionario.

Modulación del estado redox por peróxido de hidrógeno: Antes de la fecundación in vitro, los ovocitos maduros fueron incubados con diferentes concentraciones de $\mathrm{H}_{2} \mathrm{O}_{2}$ durante 1 hora: $0,50,100$ y $200 \mu \mathrm{M}$. Como el piruvato puede neutralizar el efecto de $\mathrm{H}_{2} \mathrm{O}_{2}$, este fue eliminado del medio de maduración.

Análisis estadístico: Las diferencias entre los grupos experimentales fueron analizadas mediante ANOVA de una vía para la tasa de división así como la tasa de generación de blastocitos. $\mathrm{P} \leq 0,05$ fue considerado estadísticamente significativo. Todos los análisis fueron realizados con $\mathrm{JMP}^{\circledR} 10$ (SAS Institute, Inc.).

\section{RESULTADOS}

Los ovocitos bovinos maduros que fueron incubados con diferentes concentraciones de $\mathrm{H}_{2} \mathrm{O}_{2}$ mostraron que la inducción de estrés oxidativo con 50 y $100 \mu \mathrm{M} \mathrm{H}_{2} \mathrm{O}_{2}$ no generó diferencias estadísticamente significativas en la tasa 
de división de embriones bovinos comparado con el control ( $88,7 \%$ y $83,2 \%$ respectivamente, control: $86,4 \%$ ), no así cuando fueron tratados con $200 \mathrm{MM} \mathrm{H}_{2} \mathrm{O}_{2}$, donde la tasa disminuyó significativamente a un 58,8\% (p <0,05). Al evaluar la tasa de generación de blastocitos no se observaron diferencias estadísticamente significativas cuando se utilizó $50 \mu \mathrm{M} \mathrm{H}_{2} \mathrm{O}_{2}$ comparado con el control (20,4 \% y 25,8 \%, respectivamente). Sin embargo, se observó una disminución estadísticamente significativa cuando los ovocitos fueron tratados con 100 y $200 \mu \mathrm{M} \mathrm{H}_{2} \mathrm{O}_{2}(10,7 \%$ y $3,3 \%$ respectivamente, $\mathrm{p}<0.05$ ) (Tabla I, Fig. 1).

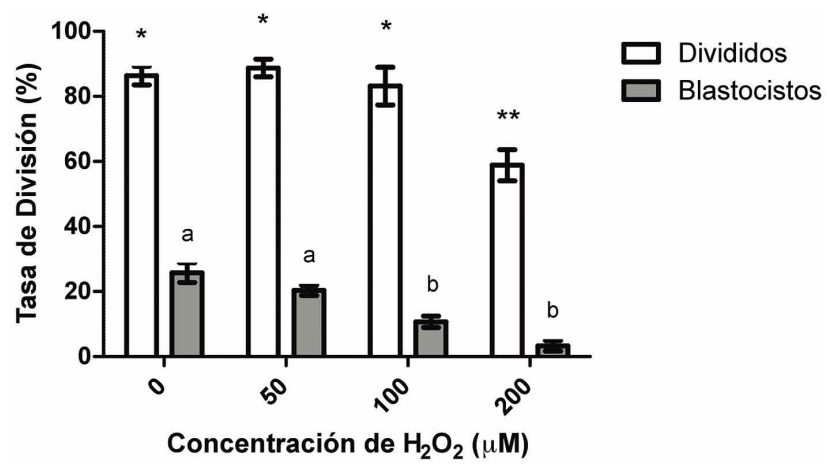

Fig. 1. Desarrollo de embriones bovinos (promedio \pm error estándar de la media) mediante fecundación in vitro luego de la modulación del estado redox por $\mathrm{H}_{2} \mathrm{O}_{2}$. Valores con diferentes superíndices o símbolos en las diferentes columnas difieren significativamente $(\mathrm{p} \leq 0,05)$.

Tabla I. Tasa de división y generación de blastocitos de embriones bovinos generados por fecundación in vitro luego de la modulación del estado oxido-reducción por peróxido de hidrógeno.

\begin{tabular}{lcccc}
\hline $\mathbf{H}_{2} \mathbf{O}_{2}$ & $\boldsymbol{n}$ & Lisados & Divididos & Blastocitos \\
\hline $0 \mu \mathrm{M}$ & 157 & 3 & $135(86,4 \pm 2,9)^{\mathrm{a}}$ & $44(25,8 \pm 3,0)^{\mathrm{a}}$ \\
$50 \mu \mathrm{M}$ & 140 & 2 & $104(88,7 \pm 2,7)^{\mathrm{a}}$ & $26(20,4 \pm 1,6)^{\mathrm{a}}$ \\
$100 \mu \mathrm{M}$ & 141 & 4 & $92(83,2 \pm 5,8)^{\mathrm{a}}$ & $16(10,7 \pm 1,7)^{\mathrm{b}}$ \\
$200 \mu \mathrm{M}$ & 114 & 13 & $48(58,8 \pm 4,8)^{\mathrm{b}}$ & $6(3,3 \pm 1,6)^{\mathrm{b}}$ \\
\hline
\end{tabular}

Los datos son presentados como promedio \pm error estándar de la media. Entre columnas, valores con diferentes superíndices difieren significativamente $(\mathrm{p} \leq 0,05)$. $\mathrm{N}=$ número de ovocitos cultivados.

\section{DISCUSIÓN}

Existe una disminución en el desarrollo o arresto en embriones mamíferos preimplantacionales cultivados in vitro cuando son comparados con los embriones que se desarrollan in vivo (Thibault, 1972; Funahashi et al., 1994). El factor más importante que contribuye a esta diferencia es la concentración de $\mathrm{O}_{2}$, debido a que en condiciones in vivo la concentración de $\mathrm{O}_{2}$ es menor (2-8 \%) que aquella utilizada para el cultivo in vitro de embriones (aproximadamente 20
\%) (Takahashi, 2012). La disminución de la concentración de $\mathrm{O}_{2}$ a un $5 \%$ en las condiciones in vitro favorece el desarrollo embrionario (Arias et al., 2014).

En este trabajo, evaluamos el efecto de las condiciones de cultivo in vitro que dan lugar a estrés oxidativo utilizando peróxido de hidrógeno a diferentes concentraciones sobre el potencial del desarrollo embrionario. En nuestro estudio, la exposición de ovocitos bovinos maduros a 50 $\mu \mathrm{M} \mathrm{H}_{2} \mathrm{O}_{2}$ no disminuyó significativamente el potencial de desarrollo embrionario de embriones bovinos, pero no logra el incremento en el número de blastocitos con una exposición similar de $1 \mathrm{~h}$ de los ovocitos maduros a 50-100 $\mu \mathrm{M}$ $\mathrm{H}_{2} \mathrm{O}_{2}$ reportado por Vandaele et al. Asimismo, la alta presión hidrostática (APH) también genera tolerancia al estrés. Ovocitos de porcinos maduros tratados con $20 \mathrm{MPa}$ (Megapascal) durante $130 \mathrm{~min}$ incrementa significativamente la tasa de generación de blastocitos $(13,1 \%)$ comparado con el grupo control luego de la vitrificación sin APH $(0 \%)$. El pre-tratamiento con APH fue eficiente para incrementar el desarrollo in vitro de ovocitos porcinos vitrificados luego de la activación mediante partenogénesis (Du et al., 2008). En blastocitos ovinos generados in vitro pre-tratados con $40 \mathrm{MPa}$ la tasa de eclosión de blastocitos tratados fue similar con los embriones no tratados (Bogliolo et al., 2011). De manera similar, blastocitos bovinos producidos in vitro sometidos a $60 \mathrm{MPa}$ durante $1 \mathrm{~h}$ y luego vitrificados, la evaluación a las 48 h demostró que los embriones eclosionados tratados incrementaron el número de células en el macizo celular interno comparado con el control sin tratamiento $(50,2 \%$ vs. $38,8 \%)$, indicando una alta calidad embrionaria. La combinación de tratamiento con APH y vitrificación resulta en un incremento en el número de células del macizo celular interno de embriones eclosionados $48 \mathrm{~h}$ post desvitrificación (Trigal et al., 2013). En espermatozoides también se ha observado un efecto positivo. Semen fresco de porcino tratado con 200, 300 o 400 bar durante 90 minutos posterior a la descongelación, la motilidad del semen fue incrementada comparada con el control (52, 56 y $56 \%$ respectivamente; control: 46 \%) (Huang et al., 2009). La alta presión osmótica también genera tolerancia a estrés en ovocitos maduros de cerdos cuando fueron tratados con 593 y 1306 mOsm, la tasa de blastocitos generados mediante "hand made cloning" fue mayor, comparado con el control sin tratamiento (64\% y $65 \%$, respectivamente; control: 45 $\%$ (Lin et al., 2009).

El mecanismo por el cual la tolerancia a estrés es inducido aún no ha sido elucidado, aunque, es posible que las proteínas de shock térmico (HSP) podrían estar involucradas (Du et al.). La suplementación del cultivo embrionario con anticuerpos anti-HSP afecta negativamente el desarrollo embrionario, observándose un incremento 
en las células apoptóticas en los blastocitos. Así, las HSP probablemente constituyen un mecanismo de protección fundamental para evitar que disminuya la sensibilidad de las células a eventos que generan daño celular, permitiendo a la célula escapar del proceso de apoptosis (Esfandiari et al., 2007). Otra molécula involucrada en la tolerancia a estrés es un factor transcripcional, el factor inducible por hipoxia (HIF-1) (Harvey, 2007).

Blastocitos ovinos expuestos a tratamientos con APH, evaluándose 8 genes involucrados en diferentes mecanismos, produce una menor expresión del gen BAX (gen proapoptosis) y OCT4 (factor de transcripción nuclear), comparados con los embriones control sin tratamiento. La disminución de BAX en los embriones tratados sugiere una mejor calidad, y coincide con la disminución del grado de picnosis. La disminución de la expresión de OCT4 podría estar relacionada a un mayor avance en la etapa del desarrollo embrionario, lo que se relacionó con un alto número de células, comparado con el control sin tratamiento (Bogliolo et al.). La tolerancia a estrés también podría estar relacionada con un incremento en las defensas antioxidantes endógenas. Se produce una abundancia en la expresión de transcritos relacionados con estrés oxidativo, superóxido dismutasa, catalasa, glutatión peroxidasa e interferón t cuando los embriones son cultivados en un sistema con elevada tensión de $\mathrm{O}_{2}$ (Corrêa et al., 2008).

En conclusión, la modulación redox de ovocitos bovinos maduros mediante $\mathrm{H}_{2} \mathrm{O}_{2}$ en dosis de $50 \mu \mathrm{M}$ no afecta la tasa de división embrionaria, así como en la tasa de generación de blastocitos. Es posible, que estos embriones resistentes al estrés oxidativo puedan tener una mayor sobrevida a procesos de criopreservación que genera altos niveles de especies reactivas de oxígeno en los embriones. No obstante, se hace necesario determinar cuáles son los mecanismos específicos involucrados en la tolerancia a estrés.

\section{AGRADECIMENTOS}

Este trabado fue financiado por Proyecto FONDECYT 1130888, CONICYT, Gobierno de Chile. P. Loren es Becaria CONICYT. Los autores agradecen al Frigorífico Temuco por la donación de ovarios de bovino.

LOREN, P.; CHEUQUEMÁN, C.; RISOPATRÓN, J.; FELMER, R.; ARIAS, M. E. \& SÁNCHEZ, R. Modulation of redox state by hydrogen peroxide in the stage of oocyte maturation: Effect on embryonic development in cattle. Int. J. Morphol., 34(2):431-435, 2016.

SUMMARY: Oxidative stress is defined as an imbalance between the production of oxidants and antioxidants. The induction of stress tolerance in oocytes leads to a better embryonic development. In cattle incubating mature oocytes with different stressors (thermal, high hydrostatic pressure, oxidative) increase the generation rate of blastocysts. The purpose of this study was to evaluate the effect of modulating the redox state increasing the oxidative stress through $\mathrm{H}_{2} \mathrm{O}_{2}$ in mature oocyte under in vitro culture conditions and its effect on the potential of embryonic development. To do this, oocytes from slaughterhouse ovaries were matured in TCM-199 medium supplemented for $22-23 \mathrm{~h}$ at $38.5^{\circ} \mathrm{C}, 5 \% \mathrm{CO}_{2}$ and humidified atmosphere. At the end of $22-23 \mathrm{~h}$, the treatments with $0,50,100$ and 200 $\mu \mathrm{M} \mathrm{H}_{2} \mathrm{O}_{2}$ were applied for $1 \mathrm{~h}$. IVF was performed co-incubating the eggs for $18 \mathrm{~h}$ with a final concentration of $1 \times 10^{6} \mathrm{sperm} / \mathrm{mL}$. The presumptive zygotes were denuded and cultured in medium KSOM-0.4\% BSA to $38.5{ }^{\circ} \mathrm{C}$ in an atmosphere of low concentration of $\mathrm{O}_{2}$ $\left(5 \% \mathrm{O}_{2}, 5 \% \mathrm{CO}_{2}\right.$ and $\left.90 \% \mathrm{~N}_{2}\right)$ and humidified atmosphere. The results show that the induction of oxidative stress by $\mathrm{H}_{2} \mathrm{O}_{2}$ produces a similar effect using a concentration of 50 and $100 \mathrm{mM}$ in the cleavage rate of embryos compared to control $(88.7 \%, 83.2 \%$ and $86,4 \%$ respectively, $\mathrm{p}>0.05)$ and decreasing significantly by using a concentration of $200 \mathrm{mM}(58.8 \%, \mathrm{p}<0.05)$. Also, $\mathrm{H}_{2} \mathrm{O}_{2}$ caused a similar effect on the rate of blastocysts with $50 \mu \mathrm{M}(20.4 \%$ vs. 25.8 control, p>0.05) but decreased significantly with 100 and $200 \mu \mathrm{M}(10.7 \%$ and $3.3 \%$ respectively, $\mathrm{p}<0.05$ ). It is possible that these embryos resistant to oxidative stress may have a higher survival in the cryopreservation processes that generating high levels of reactive oxygen species.

KEY WORDS: In vitro fertilization (IVF); Oxidative stress; Stress tolerance; Reactive oxygen species.

\section{REFERENCIAS BIBLIOGRÁFICAS}

Agarwal, A.; Said, T. M.; Bedaiwy, M. A.; Banerjee, J. \& Alvarez, J. G. Oxidative stress in an assisted reproductive techniques setting. Fertil. Steril., 86(3):503-12, 2006.

Arias, M. E.; Sanchez, R. \& Felmer, R. Evaluation of different culture systems with low oxygen tension on the development, quality and oxidative stress-related genes of bovine embryos produced in vitro. Zygote, 20(3):209-17, 2012.

Bogliolo, L.; Ariu, F.; Leoni, G.; Uccheddu, S. \& Bebbere, D. High hydrostatic pressure treatment improves the quality of in vitroproduced ovine blastocysts. Reprod. Fertil. Dev., 23(6):80917, 2011. 
LOREN, P.; CHEUQUEMÁN, C.; RISOPATRóN, J.; FELMER, R.; ARIAS, M. E. \& SÁNCHEZ, R. Modulación del estado de óxido-reducción por peróxido de hidrógeno en la etapa de maduración ovocitaria: efecto sobre el desarrollo embrionario en bovinos. Int. J. Morphol., 34(2):431-435, 2016.

Corrêa, G. A.; Rumpf, R.; Mundim, T. C.; Franco, M. M. \& Dode, M. A. Oxygen tension during in vitro culture of bovine embryos: effect in production and expression of genes related to oxidative stress. Anim. Reprod. Sci., 104(2-4):132-42, 2008.

Das, S.; Chattopadhyay, R.; Ghosh, S.; Ghosh, S.; Goswami, S. K.; Chakravarty, B. N. \& Chaudhury, K. Reactive oxygen species level in follicular fluid--embryo quality marker in IVF? Hum. Reprod., 21(9):2403-7, 2006.

Du, Y.; Pribenszky, C. S.; Molnár, M.; Zhang, X.; Yang, H.; Kuwayama, M.; Pedersen, A. M.; Villemoes, K.; Bolund, L. \& Vajta, G. High hydrostatic pressure: a new way to improve in vitro developmental competence of porcine matured oocytes after vitrification. Reproduction, 135(1):13-7, 2008.

Esfandiari, N.; Falcone, T.; Goldberg, J. M.; Agarwal, A. \& Sharma, R. K. Heat-shock proteins modulate the incidence of apoptosis and oxidative stress in preimplantation mouse embryos. Fertil. Steril., 87(5):1214-7, 2007.

Funahashi, H.; Stumpf, T. T.; Terlouw, S. L.; Cantley, T. C.; Rieke, A. \& Day, B. N. Developmental ability of porcine oocytes matured and fertilized in vitro. Theriogenology, 41(7):142533, 1994

Goto, Y.; Noda, Y.; Mori, T. \& Nakano, M. Increased generation of reactive oxygen species in embryos cultured in vitro. Free Radic. Biol. Med., 15(1):69-75, 1993.

Harvey, A. J. The role of oxygen in ruminant preimplantation embryo development and metabolism. Anim. Reprod. Sci., 98(1-2):113-28, 2007.

Huang, S. Y.; Pribenszky, C.; Kuo, Y. H.; Teng, S. H.; Chen, Y. H.; Chung, M. T. \& Chiu, Y. F. Hydrostatic pressure pretreatment affects the protein profile of boar sperm before and after freezing-thawing. Anim. Reprod. Sci., 112(1-2):13649, 2009.

Lin, L.; Du, Y.; Liu, Y.; Kragh, P. M.; Li, J.; Purup, S.; Kuwayama, M.; Zhang, X.; Yang, H.; Bolund, L. \& Vajta, G. Elevated $\mathrm{NaCl}$ concentration improves cryotolerance and developmental competence of porcine oocytes. Reprod. Biomed. Online, 18(3):360-6, 2009.

Liu, Y.; Fiskum, G. \& Schubert, D. Generation of reactive oxygen species by the mitochondrial electron transport chain. $J$. Neurochem., 80(5):780-7, 2002.

Ma, Q. Transcriptional responses to oxidative stress: pathological and toxicological implications. Pharmacol. Ther., 125(3):376-93, 2010.

Morado, S. A.; Cetica, P. D.; Beconi, M. T. \& Dalvit, G. C. Reactive oxygen species in bovine oocyte maturation in vitro. Reprod. Fertil. Dev., 21(4):608-14, 2009.
Rahman, M. B.; Vandaele, L.; Rijsselaere, T.; Zhandi, M.; Maes, D.; Shamsuddin, M. \& Van Soom, A. Oocyte quality determines bovine embryo development after fertilisation with hydrogen peroxide-stressed spermatozoa. Reprod. Fertil. Dev., 24(4):608-18, 2012.

Roy, S. C. \& Atreja, S. K. Effect of reactive oxygen species on capacitation and associated protein tyrosine phosphorylation in buffalo (Bubalus bubalis) spermatozoa. Anim. Reprod. Sci., 107(1-2):68-84, 2008.

Takahashi, M. Oxidative stress and redox regulation on in vitro development of mammalian embryos. J. Reprod. Dev., $58(1): 1-9,2012$.

Thibault, C. Some pathological aspects of ovum maturation and gamete transport in mammals and man. Acta Endocrinol. Suppl. (Copenh.), 166:59-66, 1972.

Trigal, B.; Muñoz, M.; Gómez, E.; Caamaño, J. N.; Martin, D.; Carrocera, S.; Casais, R. \& Diez, C. Cell counts and survival to vitrification of bovine in vitro produced blastocysts subjected to sublethal high hydrostatic pressure. Reprod. Domest. Anim., 48(2):200-6, 2013.

Vandaele, L.; Thys, M.; Bijttebier, J.; Van Langendonckt, A.; Donnay, I.; Maes, D.; Meyer, E. \& Van Soom, A. Short-term exposure to hydrogen peroxide during oocyte maturation improves bovine embryo development. Reproduction, 139(3):505-11, 2010.

Dirección para Correspondencia:

Raúl Sánchez Gutiérrez

BIOREN-CEBIOR

Campus Valentín Letelier

Universidad de La Frontera

Montevideo 0870

Temuco

CHILE

Email: raul.sanchez@ufrontera.cl

Recibido : 18-01-2016

Aceptado: 14-03-2016 